\title{
Epidemic Dynamics and Patterns of Plant Diseases
}

\author{
J. Segarra, M. J. Jeger, and F. van den Bosch
}

First author: Department of Agronomy and Forestry, ETSEA, University of Lleida, Av. Alcalde Rovira Roure 177, 25198 Lleida, Spain; second author: T.H. Huxley School, Imperial College at Wye, University of London, Wye, Ashford, Kent TN25 5AH, U.K.; and third author: Department of Statistics, IACR-Rothamsted, Harpenden, Hertfordshire AL5 2JQ, U.K.

Accepted for publication 27 June 2001.

\begin{abstract}
Segarra, J., Jeger, M. J., and van den Bosch, F. 2001. Epidemic dynamics and patterns of plant diseases. Phytopathology 91:1001-1010.

The general Kermack and McKendrick epidemic model (K\&M) is derived with an appropriate terminology for plant diseases. The epidemic dynamics and patterns of special cases of the K\&M model, such as the Vanderplank differential-delay equation; the compartmental healthy $(H)$, latent $(L)$, infectious $(S)$, and postinfectious $(R)$ model; and the $\mathrm{K} \& \mathrm{M}$

how $R_{0}$ and the normalized sporulation curve can be calculated from data in the literature. There are equivalences in the values of the basic reproductive number, $R_{0}$, the epidemic threshold, and the final disease level across the different models. However, they differ in expressions for the initial disease rate, $r$, and the initial infection, $Q$, because the values depend on the sporulation curve. Expressions for $r$ and $Q$ were obtained for each model and can be used to approximate the epidemic curve by the logistic equation.
\end{abstract} model with a delay-gamma-distributed sporulation curve were compared. The characteristics of the disease cycle are summarized by the basic reproductive number, $R_{0}$, and the normalized sporulation curve, $i(\tau)$. We show
Additional keywords: epidemiology, mathematical models.
Many different models and modeling approaches have been used in botanical epidemiology. These include descriptive growth curves $(23,36)$, followed by epidemic simulators $(31,37)$, to recently, models more in line with those developed in population ecology and theoretical epidemiology (32-34) for airborne and soilborne (38), fungal and viral (6) diseases.

The growth curve most widely used to describe and compare epidemic progress is the logistic model,

$$
\frac{d Y}{d t}=r Y\left[1-\left(\frac{Y}{K}\right)\right]
$$

where $Y$ represents the quantity of disease, $r$ is a disease rate parameter, and $K$ is the maximum quantity of disease or "carrying capacity." When $Y$ is measured as a proportion in disease assessments, $K=1$.

From a disease dynamics perspective, it is preferable to represent disease as the amount of infectious tissue, e.g., plant surface bearing sporulating colonies of a fungal pathogen, because this is what contributes to the progress of the disease. When disease is measured as a proportion, Vanderplank (35) adapted the logistic equation to an epidemiologically more realistic model by the incorporation of latent $(p)$ and infectious $(i)$ periods

$$
\frac{d y_{t}}{d t}=R\left(y_{t-p}-y_{t-p-i}\right)\left(1-y_{t}\right)
$$

in which $y_{t}$ is the proportion of disease at time $(t)$ including diseased tissue that is latent (preinfectious), infectious, and removed (postinfectious), and $R$ is a disease transmission rate that relates the increase in disease to the amount of infectious $\left(y_{t-p}-y_{t-p-i}\right)$ and healthy $\left(1-y_{t}\right)$ tissue. In this, we deviate from Berger (3) who excludes latent tissue from his definition of disease. Equation 2 has been used in many simulation models as the basis for predicting epidemic development.

Corresponding author: M. J. Jeger; E-mail address: m.jeger@ic.ac.uk

Publication no. P-2001-0809-01R

(c) 2001 The American Phytopathological Society
In theoretical epidemiology, compartmental models are often used with individuals classified as healthy $(H)$, latent $(L)$, infectious $(S)$, and postinfectious $(R)$. This corresponds to the more usual terminology of susceptible $(S)$, exposed $(E)$, infectious $(I)$, and removed $(R)$ used in human and animal epidemiology. These models are formulated as systems of linked differential equations $(6,13,28)$. An H-L-S-R epidemic model of a polycyclic plant disease in a closed population can be formulated as the system:

$$
\begin{aligned}
& d H / d t=-\beta S H \\
& d L / d t=\beta S H-\theta L \\
& d S / d t=\theta L-\alpha S \\
& d R / d t=\alpha S \\
& N=H+L+S+R
\end{aligned}
$$

where $\beta$ is a per capita disease transmission rate, and $\theta$ and $\alpha$ are constant rates of progress from the latent to infectious and from the infectious to postinfectious compartments, respectively.

Equations 2 and 3 fit into the same mathematical framework. In fact, if $N=1$ and $R=\beta$, the only difference lies in the distributions assumed for the latent and the infectious periods. In equation 2 , latent and infectious periods are constant and equal for all individuals, whereas in equation 3 the duration of both periods is assumed to be distributed exponentially, with rate $\theta$ the inverse of the mean length of latent $(p)$ and rate $\alpha$ the inverse of the mean length of the infectious ( $i$ ) period. The rates $\theta$ and $\alpha$ are constants in equation 3 . For both models, we can introduce a source function $g(t)$ describing spores arising from a primary infection.

An early contribution on mathematical theory of epidemics was made by Kermack and McKendrick (19) in the context of human disease. From this pioneering work, key epidemiological parameters were derived, such as the invasion criterion for disease to establish itself and the final size of the epidemic. The Kermack and McKendrick (K\&M) epidemic model has been extensively analyzed and reviewed in the mathematical literature (10). Although, applied first in animal and human epidemiology, it is of general nature and has been adapted to spatial spread of plant diseases (32-34). However, it has not been developed in its own right from 
first principles in relation to key characteristics of plant disease epidemics.

The aims of this paper are to (i) derive the general $\mathrm{K} \& \mathrm{M}$ model with an appropriate terminology for plant diseases, (ii) discuss the Vanderplank (equation 2) and the H-L-S-R (equation 3) models as special cases of the general $\mathrm{K} \& \mathrm{M}$ model, (iii) compare their qualitative dynamics, and (iv) derive an approximation to the disease rate in equation 1.

\section{THEORY AND APPROACHES}

Model. In this section, the K\&M model is derived with terminology appropriate to a foliar fungal disease that multiplies through the production and airborne dispersal of spores as the infectious propagules. The model will be based on the knowledge of the underlying mechanisms of the infection cycle. A problem in plant pathology is to specify precisely what is the individual or unit of assessment. The proper choice of unit depends on the nature of the disease. Standard measures of disease include number of diseased plants or plant organs (leafs, stalks, and buds), proportion of tissue diseased, and number or density of lesions. In what follows, we will consider the unit of disease to be a lesion and for the host a site, being the characteristic area occupied by a lesion. It is assumed that the growth of the host population and of individual lesions is insignificant and that spore dispersal is homogeneous throughout a population of healthy and diseased plants. The effect of primary inoculum is represented through setting initial conditions for the disease. The variables and parameters introduced are listed in Table 1.

Life-history characteristics. The disease is transmitted from infectious to healthy sites by a sequence of infection cycles. Each cycle consists of four phases: infection, latency, sporulation, and dispersion. When a viable spore, arriving from an external source, deposits on a healthy site, an epidemic may be initiated. If the host is susceptible and the environmental conditions favorable, the propagule will germinate, penetrate the plant surface, and establish an infection. Once an infection is established there is a latent period during which the colonization of the plant tissue takes place internally without producing spores. After this period of latency, spore production external to the plant will gradually increase, reach a peak, decrease, and eventually cease once plant resources at that site are exhausted. The rate of sporulation depends on lesion age since the time of infection $(\tau)$. Once the period of infectiousness has passed, the lesion no longer produces spores.

From the spores produced during a lesion's lifetime, only a proportion will infect a new site and start a new lesion. Spores may be deposited on the ground or escape from the canopy boundary layer. Secondly, though deposited on a healthy site, they may fail to germinate or penetrate. Once a spore successfully infects a site, another infection cycle is initiated.

The basic life-history parameters underlying the epidemic process are (i) the sporulation curve, $I(\tau)\left[N_{s p} T^{-1} N_{l e}{ }^{-1}\right]$ (this is the number of spores produced per unit of time by an infected site of age $(\tau)$ and it includes both the latent period, during which $I(\tau)=0$, and the infectious period), (ii) the probability that a spore is deposited on a particular host site, $\xi\left(N_{s t}^{-1}\right)$, and (iii) the probability that a spore landing on a healthy site initiates a new infection, $\psi\left(N_{l e} N_{s p}{ }^{-1}\right)$.

To derive parameters with a clear biological interpretation, we introduce the basic reproductive number, $R_{0}$. The biological definition of $R_{0}$ is the average number of new lesions produced by a lesion during its period of infectiousness in the early stages of the epidemic, when essentially all plants are not diseased and susceptible (24). By definition, for any sporulation curve,

$$
R_{0}=\psi \xi H_{0} \int_{0}^{\infty} I(\tau) d \tau
$$

where $H_{0}$ is the total number of healthy sites at $t=0$. It is worth noting that the expression for $R_{0}$ (equation 4 ) includes all the three lesion life-history parameters and takes into account the total number of spores. A related quantity that varies throughout the epidemic is the effective reproductive ratio (1) or again number $\left(R_{e}\right)$, equivalent to the total number of lesions produced by a lesion at any stage of the epidemic; for the K\&M model, and its special cases, $R_{e}$ decreases linearly with the proportion of healthy sites. The timing of spore production is described by the normalized sporulation curve, $i(\tau)$,

$$
i(\tau)=\frac{I(\tau)}{\int_{0}^{\infty} I(\tau) d \tau}
$$

Therefore, $R_{0}$ and $i(\tau)$ summarize the quantitative characteristics of the infection cycle, have a clear biological interpretation, and are the key components of temporal epidemic development.

Development of the model. In accordance with the previous assumptions and life-history characteristics, we will model the dynamics of a population of lesions. The rate of change of healthy sites, $H(t)$, in a population is given by

$$
\frac{d H}{d t}=-\psi \xi H(t) A(t)
$$

where $A(t)$ is the number of spores produced by the entire population of lesions per unit of time, and $\psi \xi H(t)$ represents the infection probability per spore. For the purposes of this paper, we consider the term $A(t)$ to be the sum of two categories of spores: those that result from the initial infection $g(t)$, and the spores produced by secondary infection cycles $A_{1}(t)$. In this, we follow the procedure outlined by Kermack and McKendrick (19) and several other authors $(8,26)$.

Thus,

$$
A(t)=A_{1}(t)+g(t)
$$

to calculate the total spore production, $A_{1}(t)$, we need to add the spore production of all lesions present at time, $t$. Because the population is closed, $-d H(t) / d t$ is the number of new infections per unit time, $t$, therefore $-d H(t-\tau) / d t$ is the number of lesions arising per unit of time, that at $t$ have infection age, $\tau$. Therefore,

$$
A_{1}(t)=-\int_{0}^{t} \frac{d H(t-\tau)}{d t} I(\tau) d \tau
$$

The spore production of the initial infection, $g(t)$, can take various special forms. If the epidemic is started internally at $t=0$ by $T$ lesions of age $\tau=0, g(t)$ has the form

$$
g(t)=T I(\tau)
$$

It could also be the case that spores from an external source area are deposited on the plant population. Let $h(t)$ be the number of spores deposited per unit of time and per site. In that case, the initial condition takes the form

$$
g(t)=\int_{0}^{t} \psi \xi h(t-\tau) I(\tau) d \tau
$$

Combining equations 6, 7, and 8 together, the K\&M model (19) is given by

$$
\frac{d H(t)}{d t}=\psi \xi H(t)\left[\int_{0}^{t} \frac{d H(t-\tau)}{d t} I(\tau) d \tau-g(t)\right]
$$

This integro-differential equation describes the disease progress for any sporulation curve, $I(\tau)$.

Special cases of the K\&M model. May (24) emphasized that the compartmental and Vanderplank models are limiting cases of a more general framework in which individuals move out of the 
infected compartment at a rate that explicitly depends on the elapsed time since the infection took place. We now discuss the Vanderplank (equation 2) and H-L-S-R (equation 3) models as special cases of the general K\&M model (equation 11) in which specific assumptions are incorporated into the life history of one lesion.

Vanderplank model (equation 2). That model assumes that during an infectious period of fixed duration, a lesion produces a constant number of spores per unit of time, $\gamma$. So, the sporulation curve of one lesion is represented by the block-function (Fig. 1)

$$
I(\tau)=\left\{\begin{array}{lll}
0 & \text { if } & \tau<p \\
\gamma & \text { if } & p<\tau<p+i \\
0 & \text { if } & \tau>p+i
\end{array}\right.
$$

and the normalized sporulation curve is

$$
i(\tau)=\left\{\begin{array}{lll}
0 & \text { if } & \tau<p \\
1 / i & \text { if } & p<\tau<p+i \\
0 & \text { if } & \tau>p+i
\end{array}\right.
$$

When equation 12 is substituted into equation 11 and the number of healthy sites is replaced by the number of diseased sites $Y(t)=$ $H_{0}-H(t)$, this gives the unscaled Vanderplank equation including a more general function to describe how an epidemic is initiated, $g(t)$,

$$
\frac{d Y(t)}{d t}=R\left(Y_{t-p}-Y_{t-p-i}+g(t)\right)\left(1-\frac{Y(t)}{H_{0}}\right)
$$

where $R=\psi \xi H_{0} \gamma$.

The parameter $R$ is the number of lesions produced per infectious lesion per day in a completely susceptible crop. As a lesion sporulates for exactly $i$ days in the Vanderplank model (equation 2 ), the basic reproductive number thus is

$$
R_{0}=R i
$$

equivalent to the "progeny-parent ratio" as defined by Vanderplank (35).

H-L-S-R model (equation 3). In the compartmental model, it is assumed that any lesion in its latent stage becomes infectious with a constant probability per unit of time $(\theta)$. Once infectious, the lesion produces a constant number of spores per unit of time $(\gamma)$ and has a probability per unit of time $(\alpha)$ to cease spore production.

Following Diekmann and Heesterbeek (9), we can derive the expression for the sporulation curve $I(\tau)$ from the H-L-S-R model (equation 3). If it is assumed that at $t=0$ one infection takes place and that there are no new infections after $t=0$, then equation 3 becomes

$$
\begin{array}{ll}
\frac{d L}{d \tau}=-\theta L & L(0)=1 \\
\frac{d S}{d \tau}=\theta L-\alpha S & S(0)=0
\end{array}
$$

When the two-equation system (equation 16) is solved, we obtain

$$
S(\tau)=\left(\frac{\theta}{\alpha-\theta}\right)\left(e^{-\theta \tau}-e^{-\alpha \tau}\right)
$$

Because the mean sporulation rate is $\gamma$ during the infectious period, the following sporulation curve is obtained as a special case of the K\&M model (equation 11):

$$
I(\tau)=\gamma S(\tau)=\gamma\left(\frac{\theta}{\alpha-\theta}\right)\left(e^{-\theta \tau}-e^{-\alpha \tau}\right) \quad \text { if } \tau>0
$$

TABLE 1 . Summary of principal variables and parameters

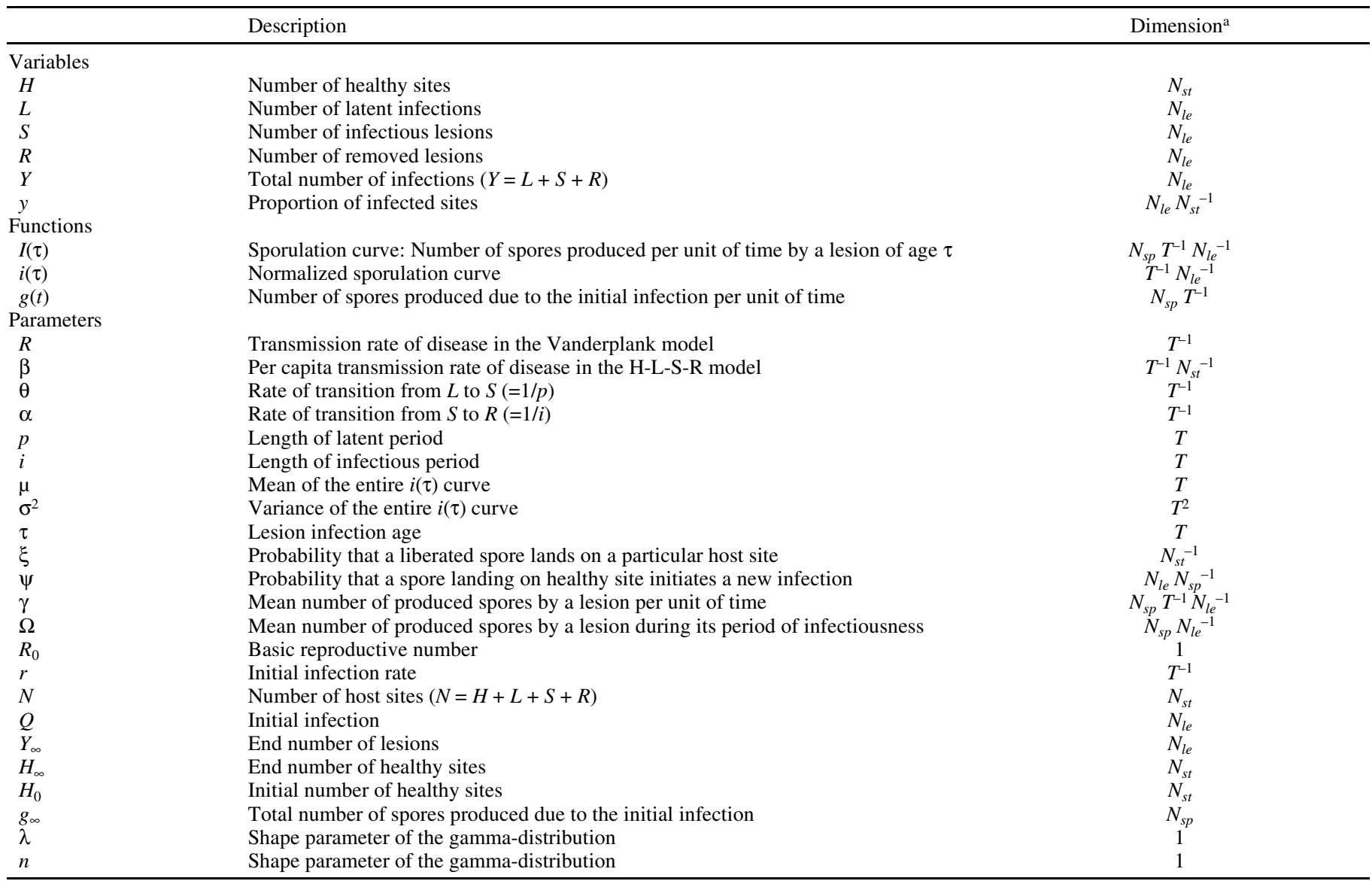

a 1 = no dimension, $N_{s t}=$ number of sites, $N_{l e}=$ number of lesions, $N_{s p}=$ number of spores, and $T=$ time. 
Equation 18 is substituted into equation 5 to give the normalized sporulation curve (Fig. 1),

$$
i(\tau)=\left(\frac{\theta \alpha}{\alpha-\theta}\right)\left(e^{-\theta \tau}-e^{-\alpha \tau}\right) \quad \text { if } \tau>0
$$

The basic reproductive number for the H-L-S-R model (equation 3) is obtained by substituting equation 18 into equation 4 and integrating to provide

$$
R_{0}=\beta H_{0}\left(\frac{1}{\alpha}\right)
$$

where $\beta=\psi \xi \gamma$.

It is worth noting that equations 15 and 20 are identical, because $R=\beta H_{0}$. Therefore, both the Vanderplank (equation 2) and H-L-S-R (equation 3 ) models lead to the same $R_{0}$ expression.

Delayed-gamma distribution. A more realistic description of the sporulation curve is obtained by the use of the delayed-gamma distribution (Fig. 1):

$$
I(\tau)=\left\{\begin{array}{lll}
0 & \text { if } & \tau<p \\
\Omega\left[\frac{\left.\lambda^{n}(\tau-p)^{n-1} e^{[-\lambda(\tau-p)]}\right]}{\Gamma(n)}\right] & \text { if } \quad \tau>p
\end{array}\right.
$$

where $\Omega$ is equivalent to $\gamma i$ in the Vanderplank model, $p$ is the latent period, and $\lambda$ and $n$ are shape parameters of the gamma distribution. This form of $I(\tau)$ has been useful to describe the sporulation curve of plant diseases such as stripe rust of wheat and downy mildew of spinach (32). The disadvantage of this description is that it is only possible to rewrite the K\&M model (equation 11 ) as a set of (delay) differential equations when $n$ is an integer, but it can be integrated numerically, when $n$ is not an integer.

\section{RESULTS}

Epidemic development. Two key aspects of the disease development are the epidemic threshold and the final size of the epidemic. Both are directly related with the basic reproductive number, $R_{0}$, but are independent of the shape of the sporulation curve, $i(\tau)$.

Epidemic threshold. According to the threshold phenomenon, the basic reproductive number, $R_{0}$, determines whether an epidemic will occur from an initial infection. For early exponential increase of the disease, it is necessary that $R_{0}>1$. If $R_{0}>1$, each lesion results in more than one lesion, but if $R_{0}<1$, each lesion

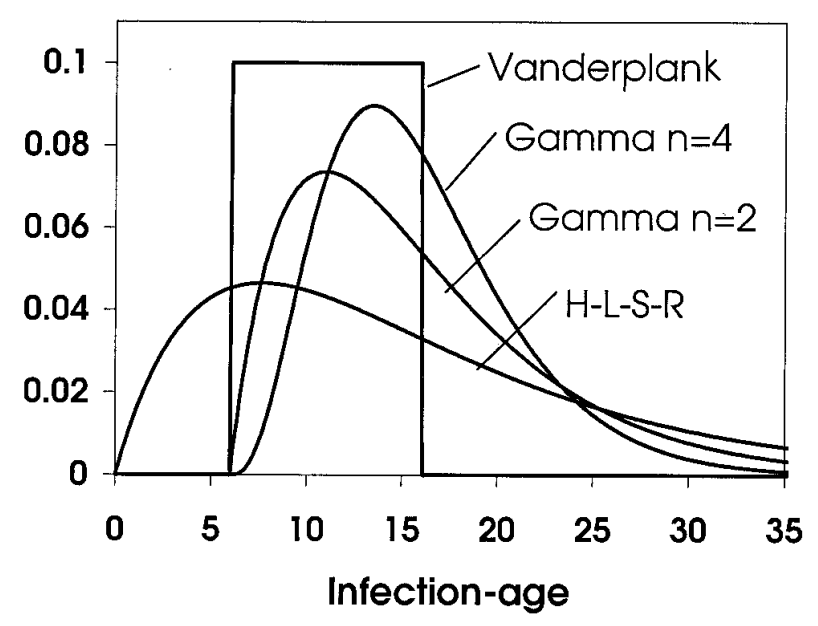

Fig. 1. Normalized sporulation curves, $i(\tau)$. Parameters values are $p=6$ and $i=10$. Shape parameters of the delayed-gamma distribution are (i) $n=2$ and $\lambda=2 / i$, and (ii) $n=4$ and $\lambda=4 / i$. The average duration of the infection cycle is $p+i$ in all four distributions. does not replace itself with another lesion and an epidemic does not occur (17).

This result can readily be derived for the H-L-S-R model (equation 3 ). When the second and third equation of the model (equation 3) are added, this gives $d(L+S) / d t=\beta S H-\alpha S$. At the start of epidemic, $H=H_{0}$, thus $d(L+S) / d t=\left(\beta H_{0}-\alpha\right) S$. Therefore, if $\beta H_{0}(1 / \alpha)<1$, there is a progressive decrease in the number of latent plus infectious sites and only a small and limited increase in removed sites results, but if $\beta H_{0}(1 / \alpha)>1$, an epidemic occurs. This inequality corresponds to the same expression of epidemic threshold $R i>1$ obtained by Vanderplank for equation 2 (35).

Final size of the epidemic, $\boldsymbol{Y}_{\infty}$. In the course of the epidemic, because there is no influx of new healthy sites and all lesions eventually cease to produce spores, the development of the disease eventually ceases. The dynamics of equations 2,3 , and 11 tend asymptotically to an equilibrium, at which point all disease is "removed" or postinfectious (Fig. 2).

The general final size equation can be obtained from the K\&M epidemic model (equation 11). We follow the derivation given by Metz (25). Division of both sides of equation 11 by $H(t)$ gives

$$
\frac{1}{H(t)} \frac{d H}{d t}=\psi \xi \int_{0}^{t} \frac{d H(t-\tau)}{d t} I(\tau) d \tau-\psi \xi g(t)
$$

Integration of both sides with respect to $t$ gives

$$
\ln \left[\frac{H\left(t_{1}\right)}{H_{0}}\right]=\psi \xi \int_{0}^{t_{1}} \int_{0}^{t} \frac{d H(t-\tau)}{d t} I(\tau) d \tau d t-\psi \xi \int_{0}^{t_{1}} g(t) d t
$$

Now,

$$
\begin{aligned}
\int_{0}^{t_{1} t} \frac{d H(t-\tau)}{d t} I(\tau) d \tau d t & =\int_{0}^{t_{1} t_{1}} I(\tau) \frac{d H(t-\tau)}{d t} d t d \tau \\
& =\int_{0}^{t_{1}} I(\tau)\left[H\left(t_{1}-\tau\right)-H_{0}\right] d \tau \\
& =\int_{0}^{t_{1}} I(\tau) H\left(t_{1}-\tau\right) d \tau-H_{0} \int_{0}^{t_{1}} I(\tau) d \tau
\end{aligned}
$$

When equation 24 is substituted into equation 23 and replacing $t$ for $t_{1}$, we arrive at

$$
\ln \left[\frac{H(t)}{H_{0}}\right]=\psi \xi \int_{0}^{t} I(\tau) H(t-\tau) d \tau-\psi \xi H_{0} \int_{0}^{t} I(\tau) d \tau-\psi \xi \int_{0}^{t} g(t) d t
$$

Finally, we take the limit as $t \rightarrow \infty$ and insert the $R_{0}$ expression from equation 4 to give the final value equation:

$$
1-\frac{H_{\infty}}{H_{0}}=1-e^{-\left[R_{0}\left(1-\frac{H_{\infty}}{H_{0}}\right)+\psi \xi g_{\infty}\right]} \quad \text { where } \quad g_{\infty}=\int_{0}^{\infty} g(t) d t
$$

$H_{\infty}$ is the final number of healthy sites, and $g_{\infty}$ is the total number of spores produced due to the initial infection. The final number of sites that have been infected during the course of the epidemic is $Y_{\infty}=H_{0}-H_{\infty}$.

We also rewrite equation 26 in terms of the fraction of sites infected. Suppose as in equation 9, that the epidemic is started internally at $t=0$ by $T$ infected sites, then $y_{0}=T / N$ and $y_{\infty}=Y_{\infty} / N$, where $N=H_{0}+T$ is the total number of sites. For $T<<H_{0}$, substituting these terms into equation 26 and rearranging gives

$$
y_{\infty}=1-\left(1-y_{0}\right) e^{-\left[\frac{R_{0}}{1-y_{0}}\left(y_{\infty}-\Psi \xi g_{\infty} y_{0}\right)\right]}
$$

If we make assumptions on the particular form of the initial spore production, $g(t)$, and on whether or not to incorporate the initial 
number of lesions in the total population, we arrive at various forms of the final size equation.

The asymptotic result for the Vanderplank model (equation 2) was given by Vanderplank (36) and has been derived formally by Jeger and van den Bosch (17) and Swinton and Anderson (30):

$$
y_{\infty}=1-\left(1-y_{0}\right) e^{-R_{0}\left(\frac{y_{\infty}}{1-y_{0}}\right)}
$$

where $y_{\infty}$ is the asymptotic proportion of disease, and $y_{0}$ is the initial proportion of disease.

In the case of the H-L-S-R model (equation 3), when the first equation is divided by the fourth equation, this gives

$$
\frac{d H}{d R}=-\beta H\left(\frac{1}{\alpha}\right)
$$

Separation of variables and integration, assuming the initial conditions $\left(H_{0}, 0, S_{0}\right.$, and 0$)$ and asymptotic values as $t \rightarrow \infty\left(H_{\infty}, 0,0\right.$, and $R_{\infty}$ ) yields the same implicit expression in equation 28 , where $y_{\infty}\left(=R_{\infty} / N\right)$ represents the fraction of the population that becomes diseased during the course of the epidemic, and $y_{0}\left(=S_{0} / N\right)$ is the initial proportion of disease arising from the primary infection.

These results for the final size of the epidemic (equations 27 and 28) can be simplified to the same expression, and thus the same value shown in Figure 2. For small initial levels of disease $\left(S_{0}<<N\right.$ and $\left.H_{0} \approx N\right)$, the final size equation is given by Swinton and Anderson (30):

$$
y_{\infty}=1-e^{-R_{0} y_{\infty}}
$$

where $y_{\infty}=1-H_{\infty} / N$.

The disease progress curve and the initial disease rate, $r$. In the initial phase of the epidemic, when the number of lesions is very small, disease eventually grows exponentially due to the absence of density dependence (no limitation on sites available for infection). In this exponential phase, the number of infected sites is given by

$$
Y(t)=Q e^{r t}
$$

where $r$ is the so-called rate of natural increase, and $Q$ is a measure of the initial level of infection.

For a small number of lesions, the K\&M model (equation 11) can be approximated by a linearized equation in which the number of healthy sites, $H(t)$, is replaced by the number of healthy individuals at the start of the epidemic, $H_{0}$. When equation 31 is substituted into the linearized K\&M model, we obtain

$$
\frac{d}{d t}\left(Q e^{r t}\right)=\psi \xi H_{0} \int_{0}^{\infty} \frac{d}{d t}\left(Q e^{r(t-\tau)}\right) I(\tau) d \tau
$$

We then integrate equation 32 with respect to $t$ and divide both sides by $Q e^{r t}$ to give

$$
1=R_{0} \int_{0}^{\infty} e^{-r \tau} i(\tau) d \tau
$$

the so-called characteristic equation from which $r$ can be calculated. If the disease life-history characteristics $R_{0}$ and $i(\tau)$ are known, we can calculate $r$ from equation 33 .

To compare the initial disease rate $(r)$ across the different models, we equate mean latent $(p)$ and mean infectious $(i)$ period in each of the models. In this way, for the Vanderplank model (equation 2) equations 13 and 15 are substituted into equation 33 and the integral is evaluated to obtain the following implicit expression for $r$ in terms of the parameters:

$$
r=\frac{R_{0}}{i}\left(e^{-r p}-e^{-r(p+i)}\right)
$$

An equivalent expression was already obtained by Vanderplank (35) for the initial phase of the epidemic. When the life-history characteristics (equations 19 and 20) are substituted into equation 33, an explicit expression for the initial disease rate of the H-L-S-R model (equation 3 ) is obtained

$$
r=\sqrt{m^{2}+\frac{1}{p i}\left(R_{0}-1\right)}-m \quad \text { where } \quad m=\frac{p+i}{2 p i}
$$

where we used the relations $p=1 / \theta$ and $i=1 / \alpha$, as explained previously.

We calculate $r$ for the delayed-gamma distribution (equation 21) using the examples of $n=2$ and $n=4$ for which the $i(\tau)$ distributions are shown in Figure 1. The mean infectious period of equation 21 is $i=n / \lambda$ and accordingly we take $\lambda=n / i$. The initial disease rate $(r)$ of a delayed-gamma-distributed sporulation curve with $n=2$ and $\lambda=2 / i$ is given by

$$
1=R_{0} e^{-r p}\left(\frac{1}{1+\frac{r i}{2}}\right)^{2}
$$

and with $n=4$ and $\lambda=4 / i$, it is given by

$$
1=R_{0} e^{-r p}\left(\frac{1}{1+\frac{r i}{4}}\right)^{4}
$$

To illustrate more clearly the relationships among $R_{0}, p, i$, and the initial disease rate $(r)$, a large number of solutions of the equations 34 to 37 are shown in Figure 3. The initial disease rate is rescaled to $r^{*}=r p$ so that time is measured in latent periods. Figure 3 can be used to obtain an approximation of the initial disease rate $\left(r^{*}\right)$ in relation to $R_{0}$ and $i / p$ values and to compare these rates between the different models.

For values of $R_{0}$ and $i / p$ giving low $r^{*}$ values, the Vanderplank model (equation 2) (Fig. 3A) has a faster disease progression, whereas at high $r^{*}$ values, the H-L-S-R model (equation 3) (Fig. 3B) has a faster disease progression. In the compartmental model (equation 3), lesions become infectious faster than in the Vanderplank model (equation 2); however, fewer spores are liberated soon after the start of the sporulation. Both effects are

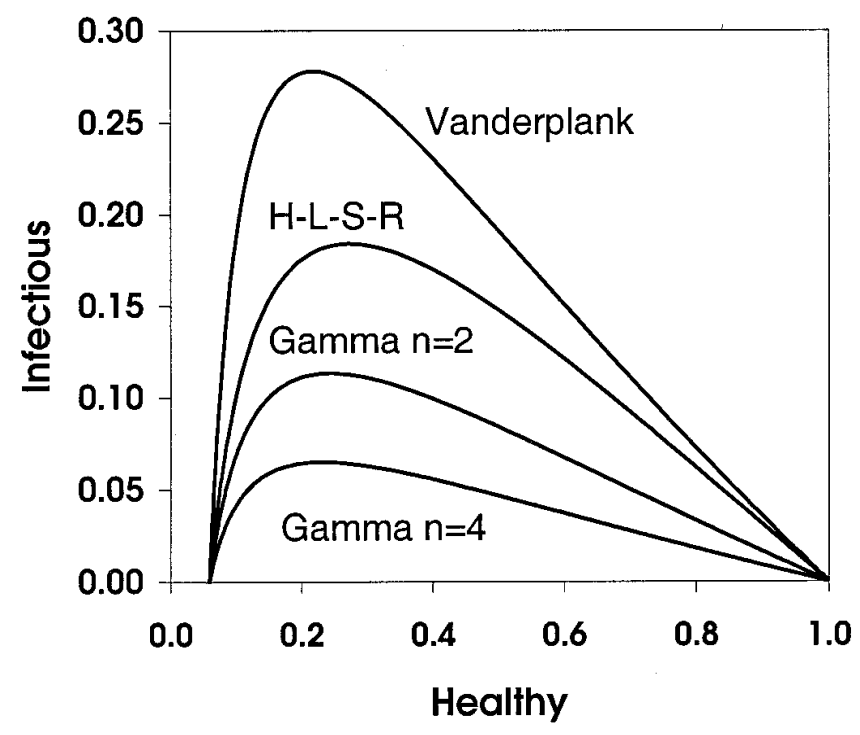

Fig. 2. Proportion of hosts that are healthy or infectious in the phase plane. Parameter values are $R=0.3, \beta=0.3 / N, \Omega=3, p=6$, and $i=10$. Initial conditions are as follows: $H_{0} / N=0.999, S_{0} / N=0.001$. Parameters are explained in Table 1. 
present and which one prevails depends on the specific parameter values. At low disease rates, the effect of the fast liberation of all spores in the Vanderplank model is more important, whereas at high disease rates, the effect of some lesions becoming infectious immediately in the compartmental model is more important.

The delayed-gamma-distributed sporulation curve leads to a reduced initial disease rate in relation to both the Vanderplank (equation 2) and H-L-S-R (equation 3) models (Fig. 3). This is because the delayed-gamma-distributed sporulation curve delays the start of sporulation of the lesions by comparison with the H-L-S-R (equation 3) model or reduces the rate at which lesions become infectious by comparison with the Vanderplank (equation 2) model (Fig. 1). In the same way, the gamma distribution with $n=$ 4 (Fig. 3C) gives a smaller $r^{*}$ than the gamma distribution with $n=2$ (Fig. 3D).

A model for the normalized sporulation curve, $i(\tau)$, of downy mildew (Peronospora farinosa (Fr.) Fr. f. sp. spinaciae Byford) on spinach (Spinacia oleracea L.) was fitted to experimental data by
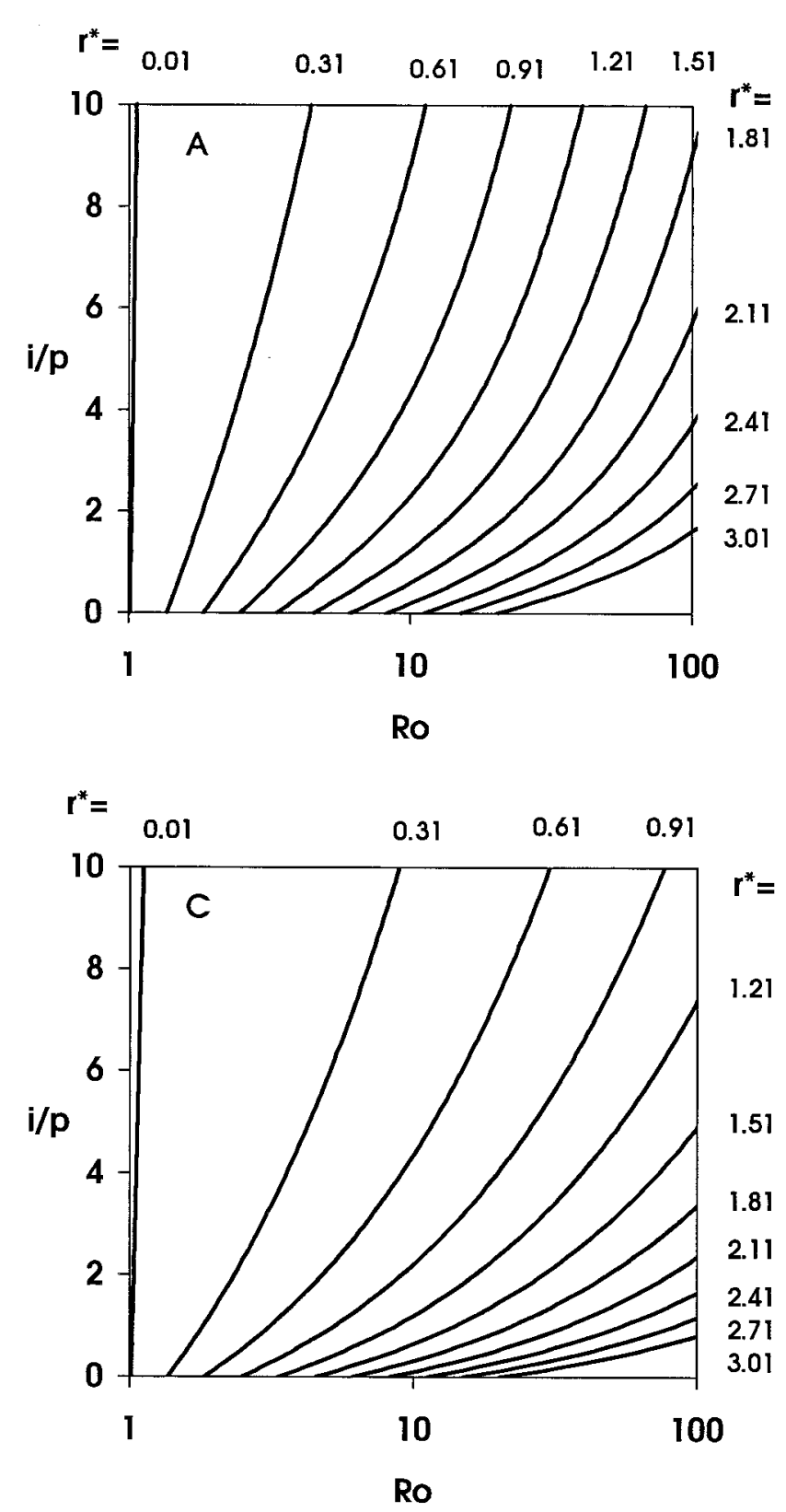

van den Bosch et al. (32). The best fit to sporulation data was given by a delayed-gamma distribution with $p=7.0$ days, $n=3.1$, and $\lambda=0.9$. The basic reproductive number, $R_{0}$, measured directly in field plots, was as estimated $R_{0}=3.2$.

When these parameter values are substituted into equation 33 , the initial disease rate is calculated as $r=0.113$ day $^{-1}$. If we assume the same mean infectious period ( $i=3.44$ days) for the different sporulation curves, we obtain for (i) the block-function, $r=0.134$ day $^{-1}$ (equation 34); (ii) the compartmental model, $r=$ 0.155 day $^{-1}$ (equation 35); (iii) the delayed-gamma distribution with $n=2, r=0.115$ day $^{-1}$ (equation 36); and (iv) the delayedgamma distribution with $n=4, r=0.113$ day $^{-1}$ (equation 37). The value of the initial disease rate clearly depends on the shape of the sporulation curve, $i(\tau)$, even when latent period and mean infectious period are numerically the same.

Where the basic reproductive number, $R_{0}$, cannot be obtained directly, equations 33 to 37 can be used to estimate $R_{0}$, provided $r$ is known. Van den Bosch et al. (32) characterized the normalized
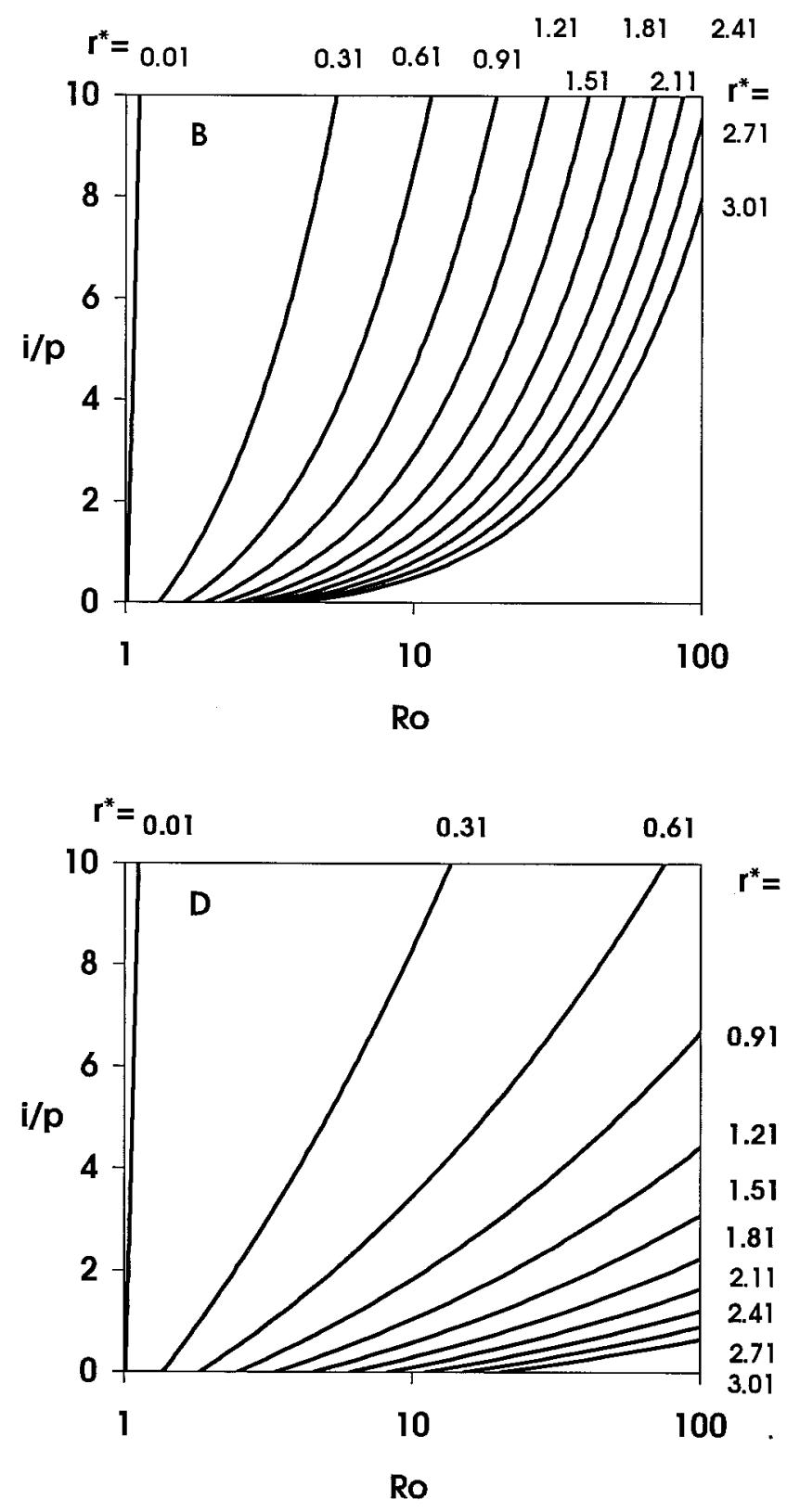

Fig. 3. Relationships of values of $R_{0}$ (basic reproductive number) and $i / p$ (infectious period/latent period) to $r^{*}$ (the initial disease rate, $r$, is rescaled so that $r^{*}=$ $r p$ implies time measured in latent periods). A, Vanderplank model; and $\mathbf{B}$, compartmental H-L-S-R model. C, Delayed-gamma-distributed sporulation curve with shape parameters $n=2$ and $\lambda=2 / i$. D, Delayed-gamma-distributed sporulation curve with shape parameters $n=4$ and $\lambda=4 / i$. For any combination of parameter values $\left(p, i\right.$, and $\left.R_{0}\right)$, the approximated $r^{*}$ value can be obtained for each model and compared among them. 
sporulation curve, $i(\tau)$, of stripe rust (Puccinia striiformis West.) on wheat (Triticum aestivum L.) with a delayed-gamma distribution with parameter values $p=10$ days, $n=3.0$, and $\lambda=0.2$. An initial disease rate was determined experimentally as $r=0.20$ day $^{-1}$. Estimates of $R_{0}$ are then obtained with (i) equation $33, R_{0}=59$; (ii) equation $34, R_{0}=23$; (iii) equation $35, R_{0}=12$; (iv) equation $36, R_{0}=46$; and (v) equation $37, R_{0}=69$. These differences again reinforce the major influence of the shape of $i(\tau)$ on values of $R_{0}$ estimated from the initial disease rate.

An approximation formula for $\boldsymbol{r}$. Any particular disease epidemic can be modeled if $R_{0}$ and the appropriate form of $i(\tau)$ are known. However, measurements on sporulation curves are laborious and are often only possible with low accuracy. In such situations, it would be useful to have an approximation for $r$ in terms of the global characteristics of $i(\tau)$, such that arbitrary judgements on the precise description of $i(\tau)$ are unnecessary. Metz and Diekmann (26) developed a useful approximation formula. We take logarithms in both sides of equation 33 to give

$$
0=\ln R_{0}+\ln \int_{0}^{\infty} e^{-r \tau} i(\tau) d \tau
$$

The second term on the right side is the so-called cumulant generating function of $i(\tau)$. We use the Taylor expansion of the cumulant generating function and the relation between cumulant and moments as given by Kendall and Stuart (18) to give

$$
0=\ln R_{0}-\mu r+\frac{1}{2} \sigma^{2} r^{2}+\ldots
$$

where $\mu$ and $\sigma$ are the mean and the standard deviation of the sporulation curve.

We already know that $r=0$ for $\ln R_{0} \leq 0$. Therefore, we expand $r$ as a power series in $\ln R_{0}$. To this end, we substitute $r=a_{1} \ln R_{0}+$ $a_{2}\left(\ln R_{0}\right)^{2}+\ldots$ in equation 39 to arrive at

$$
0=\left(\ln R_{0}\right)\left(1-\mu a_{1}\right)+\left(\ln R_{0}\right)^{2}\left(-\mu a_{2}+\frac{1}{2} \sigma^{2} a_{1}^{2}\right)+\ldots
$$

Because $\ln R_{0}$ is not equal to zero, the terms in brackets with the coefficients $a_{i}$ must be zero. Therefore, $a_{1}=1 / \mu$ and $a_{2}=$ $(1 / 2)(1 / \mu)\left(\sigma^{2} / \mu\right)$ and when these terms are substituted in the power expansion, stopping at $i=2$, we obtain the following approximation formula:

$$
r \approx \frac{\ln R_{0}}{\mu}\left[1+\frac{1}{2}\left(\frac{\sigma}{\mu}\right)^{2} \ln R_{0}\right]
$$

The $\mu$ and $\sigma$ values can be calculated from measurements of $i(\tau)$ to obtain an approximate $r$ value. Figure 4 shows the accuracy of the approximation formula (equation 41) to predict the $r$ value of the Vanderplank model (equation 34). For the more realistic forms of $i(\tau)$, the approximation formula performs better than the blockfunction of the Vanderplank model. If $\mu, \sigma$, and $R_{0}$ are calculated from experimental measurements of sporulation, Figure 4 can be used to judge the accuracy of the approximation. If data fall below the 5\% line, the approximation formula will give us $r$ values that deviate maximally $5 \%$ from the true value.

Initial level of infection, $\boldsymbol{Q}$. To calculate the initial exponential increase in the number of lesions, it is necessary to determine the initial level of infection $(Q)$. Usually, this would be determined by fitting the model to the epidemic data. If, however, we have information on the initial number of lesions, what $Q$ value provides the right approximation to match the initial rate of increase of the epidemic?

If the epidemic, for example, is initiated with $T$ lesions of age zero, we are tempted to use $Q=T$, but this is not the correct choice. Sporulation is not instantaneous because it starts only after the latent period and continues only for a limited period. The co- horts (lesions of the same age class) are, to some extent, separated during the first few generations. In the course of time, the generations increasingly overlap until the epidemic settles into steady exponential growth.

The important analytic contributions to this situation were made by Caole (5) and by Keyfitz (20,21), who showed that $Q$ depends on the initial number of lesions $(T)$ and on the normalized sporulation curve, $i(\tau)$. Using the notation introduced in this paper,

$$
Q=\frac{1}{r} \frac{\int_{0}^{\infty} e^{-r t} g(t) d t}{\int_{0}^{\infty} \tau e^{-r \tau} I(\tau) d \tau}
$$

Assume, for example, that the epidemic is started with $T$ freshly established lesions, so $g(t)=T I(\tau)$. Then, for Vanderplank model (equation 2), when the block function $I(\tau)$ (equation 12) is substituted into equation 42 and the integral is evaluated, we find

$$
Q=\frac{T}{r} \frac{1-e^{-r i}}{p+\frac{1}{r}-e^{-r i}\left(\frac{1}{r}+p+i\right)}
$$

The initial condition for the H-L-S-R model (3) is found by substituting equation 18 into equation 42 to give

$$
Q=\frac{T}{r} \frac{\alpha-\theta}{\frac{r+\alpha}{r+\theta}-\frac{r+\theta}{r+\alpha}}
$$

For the delayed-gamma distribution with $n=2$ and $\lambda=2 / i$, we substitute equation 21 into equation 42 to give

$$
Q=\frac{T}{r} \frac{1}{p+\{2 /[r+(2 / i)]\}}
$$

and with $n=4$ and $\lambda=4 / I$,

$$
Q=\frac{T}{r} \frac{1}{p+\{4 /[r+(4 / i)]\}}
$$

In Figure 5, we see that both the Vanderplank (equation 43) and H-L-S-R (equation 44) expressions for $Q$ are good descriptors of the average initial increase in the number of diseased sites, at least for this particular initial condition.

If, as in Figure 5, we assume $T=1$, then equations 43 to 46 can be used to obtain estimates of $Q$ from field data. For example, for

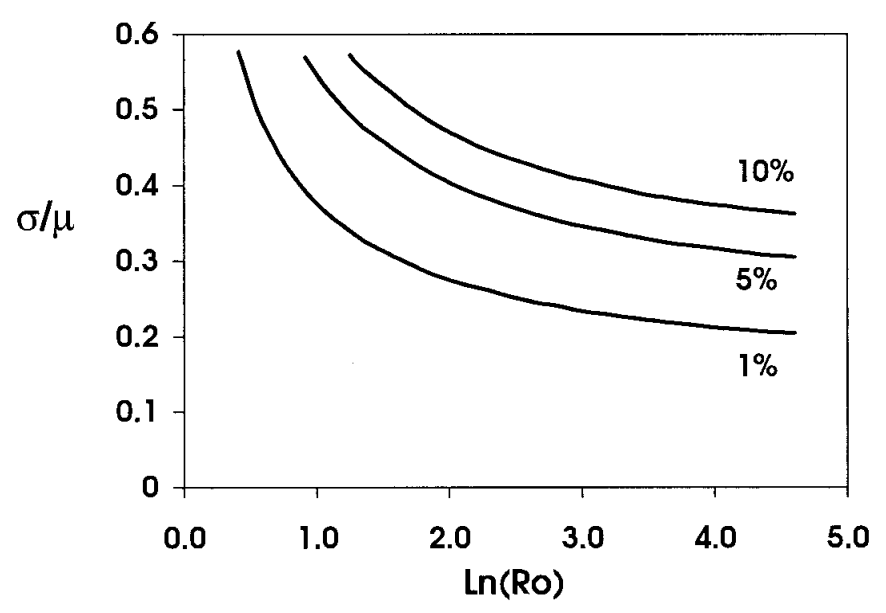

Fig. 4. Accuracy of the approximation formula (equation 41) to predict the disease rate $(r)$. Parameters are explained in Table 1. For values that fall below a given percent error level, the approximation formula gives $r$ values that deviate maximally at this level from the true value. 
the potato blight epidemic depicted by Vanderplank (Fig. 4.1, curve B for literature citation 35), we calculate $Q=0.49,1.04$, 0.45 , and 0.44 for equations 43 to 46 , respectively. The H-L-S-R model (equation 44) is the one in which $Q$ is closest to $T$ as would be expected from the shape of the sporulation curve.

Relation with the logistic equation. If the epidemic is described by the logistic model (equation 1), the value of the parameter $R$ in equation 2 varies with time and its evolution depends on the value of the parameters $(14,35)$. However, at constant values of $R$ together with certain combinations of parameters, trajectories very close to the logistic curve are obtained. Metz (25) showed that the logistic equation, suitably parametized, is an approximation to the K\&M model (equation 11). For this, we must include the parameter values that are directly related to the epidemic models. The logistic equation based on the parameters of the $\mathrm{K} \& \mathrm{M}$ model is

$$
\frac{d Y}{d t}=r Y\left[1-\left(Y / Y_{\infty}\right)\right] \quad \text { and } \quad Y(0)=Q
$$

where $Y_{\infty}$ corresponds to the final disease in an uninterrupted epidemic.
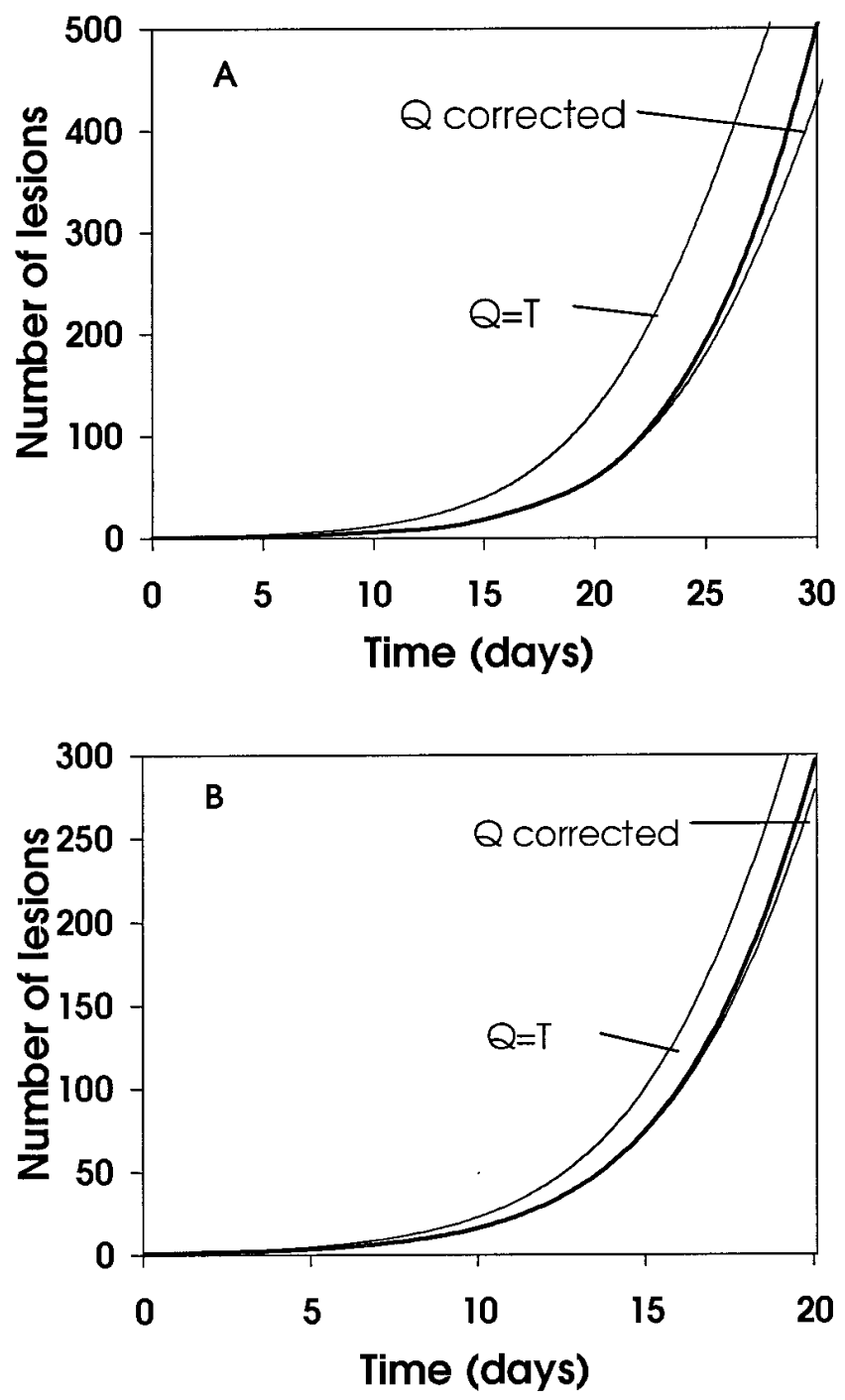

Fig. 5. Initial exponential phase of A, Vanderplank model (equation 2) compared with the exponential curve using the disease rate $(r)$ value (equation 34) with the initial infection either $Q=T$ or corrected as calculated from equation 43; and $\mathbf{B}, \mathrm{H}-\mathrm{L}-\mathrm{S}-\mathrm{R}$ model (equation 3 ) compared with the exponential curve using the disease rate $(r)$ value (equation 35 ) with the initial infection either $Q=T$ or corrected as calculated from equation 44 . Parameters values are $N=1,000, T=1, R=1.2, \beta=1.2 / N, p=6$, and $i=10$.
From the disease life-history characteristics $R_{0}$ and $i(\tau)$, we can obtain values for the parameters $r, Q$, and $Y_{\infty}$. When these values are substituted into equation 47, we obtain an approximation to the epidemic curve by the logistic equation. Epidemic curves of the Vanderplank model (equation 2) can be approximated by the logistic equation (equation 47) using the equations 34, 43, and 26 for $r, Q$, and $Y_{\infty}$, respectively (Fig. 6A). An approximation of the epidemic curves of the H-L-S-R model (equation 3 ) is obtained by substituting $r$ (equation 35), $Q$ (equation 44), and $Y_{\infty}$ (equation 26) into equation 47 (Fig. 6B). In the same way, an approximation to the K\&M model (equation 11) with a delay-gamma-distributed sporulation curve can be obtained. These logistic curves have the same initial rates and final disease levels, though there is some deviation in disease trajectories during the mid-epidemic phase.

\section{DISCUSSION}

The K\&M model (equation 11) provides a framework to analyze the dynamics of a plant disease within a season. For a sporulating lesion with an approximately uniform sporulation per day during a lesion's infectious period, a block function can be
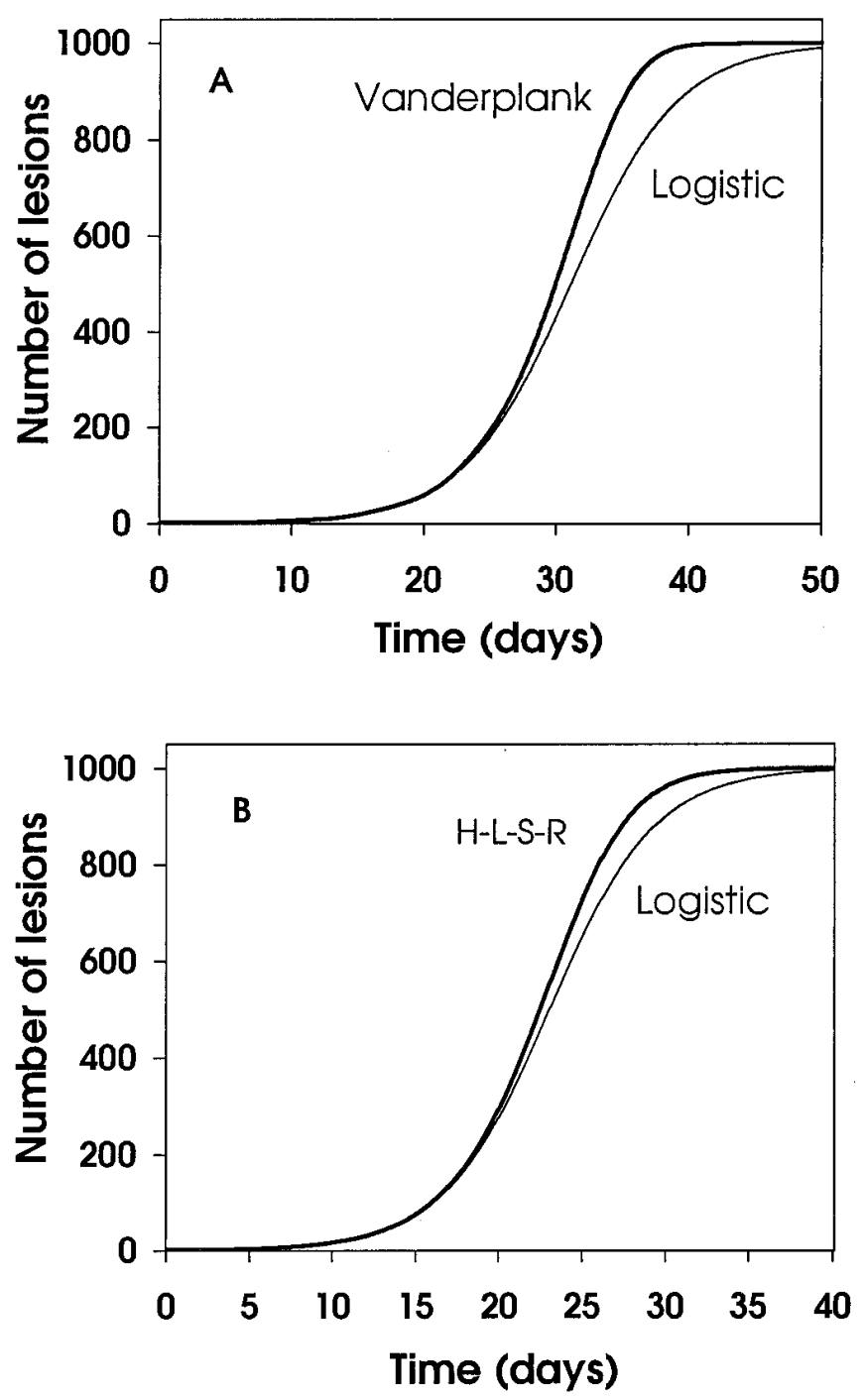

Fig. 6. Comparison of disease progress for A, the Vanderplank (equation 2) and logistic (equation 47) models with parameter values of $r, Q$, and $Y_{\infty}$ calculated from the Vanderplank model; and B, the H-L-S-R (equation 3) and logistic (equation 47) models with parameter values of $r, Q$, and $Y_{\infty}$ calculated from the H-L-S-R model. Parameters values are $N=1,000, T=1$, $R=1.2, \beta=1.2 / N, p=6$, and $i=10$. 
used as in the Vanderplank model (equation 2). For diseases that have a single peak in spore production per day, epidemic development can be modeled by the K\&M model with a delayed-gammadistributed sporulation curve (equation 21) or by the compartmental model with an exponentially distributed latent period (equation 3). Other forms for the spore production curves can be useful. For example, in an even simpler representation, several common diseases have been shown to follow an approximately triangular pattern used by Leonard and Mundt (22) to calculate the initial disease rate.

The key epidemiological (composite) parameter is the basic reproductive number, $R_{0}$, which predicts most qualitative characteristics of the host-pathogen system dynamics (17) and can be used to devise and assess the efficacy of disease control measures (24). Disease management is concerned with a reduction in $R_{0}$ because this value is closely related with the final disease level in long-term dynamics (1). Other advantages of using $R_{0}$ to characterize an epidemic is that it has a clear biological significance, its value is independent of the form of the model used, and it is dimensionless and thus directly comparable across pathosystems (27). One of the problems in fitting growth curves to epidemic data is that the values of the estimated parameters for different functions are not directly comparable (4). The use of $R_{0}$ to characterize a pathosystem or an epidemic avoids this problem.

For some fungal diseases, the parameter $R_{0}$ can be directly measured. Data on sporulation per lesion per day, duration of latent and infectious periods, and infection efficiency are relatively easy to obtain in controlled experiments $(22,29,32)$. It is more difficult to quantify the deposition probability for a healthy site. In this case, $R_{0}$ can be estimated indirectly by means of (i) the initial disease rate $(r)$, provided the correct form of the sporulation curve is known, (ii) the final disease level, or (iii) the adjusting of the model to the empirical disease progress data (12).

The parameter $R_{0}$ also determines how fast disease will spread in a population previously unexposed to that pathogen. The ordering of $R_{0}$ and $r$ is not always identical. For example, a disease may have a large $R_{0}$ but a small $r$ if the disease has a long latent period. In that sense, an epidemiological parameter used extensively by plant pathologists to summarize the epidemic curve and to evaluate management options is the initial disease rate, $r$ (35). This parameter becomes crucial to determine the speed of epidemic development and severity when transient disease progress is interrupted by, for example, crop harvest. As we have shown, the value of $r$ could be determined from the appropriate sporulation curve, $I(\tau)$, or at least approximated from the mean $(\mu)$ and the standard deviation $(\sigma)$ of the sporulation curve. However, measurements of the sporulation curve are laborious and are often not obtained and, in such cases, $r$ is estimated by fitting the model to epidemic data.

The models discussed above represent a more realistic description of the progress of disease than do growth functions. From the life-history characteristics $I(\tau), \xi$, and $\psi$, an infinite number of epidemic curves can be generated. However, none of those curves approaches the pattern of the Gompertz function, that is displacement of the epidemic peak toward the left. Nevertheless, it seems that a large number of plant epidemics are fitted better by the Gompertz equation than the logistic $(2,11)$. In this sense, other mechanisms are necessary to explain the Gompertz-type curve, such as heterogeneity in disease transmission, the growth of the crop, and variation in the susceptibility of the host population with time.

Nevertheless, the models can be used to generate disease development that approximates very closely to the form of the logistic curve. Similarly, if exact logistic growth is assumed, the value of $R$ of the Vanderplank model (equation 2) decreases to a minimum value before increasing indefinitely at the end of the epidemic (14). From a practical point of view, the parameter $R$ can be estimated throughout the epidemic period (7) whether the restrictions on the combinations of the parameters determined by
Jeger (14) are met or not. In fact, these restrictions only indicate whether the minimum value of $R$ corresponds to the beginning of the epidemic or occurs at a point during the evolution of the disease. The Vanderplank model (equation 2) has been much used in developing computer simulations of plant disease epidemics (3). Recently, the use of linked differential equation systems to model epidemics and to evaluate control strategies has increased $(6,16$, 38 ). Use of these more flexible models allows linkages with theoretical developments in human and animal epidemiology and readily allows the introduction of new biological details. The challenge for plant epidemiology is to combine these theoretical developments with experimental approaches (15).

\section{ACKNOWLEDGMENTS}

This work was supported by a research grant from the Departament d'Universitats, Recerca i Societat de la Informació of Generalitat de Catalunya to J. Segarra. IACR-Rothamsted receives grant-aided support by the Biotechnology and Biological Sciences Research Council.

\section{LITERATURE CITED}

1. Anderson, R. M., and May, R. M. 1991. Infectious Diseases of Humans: Dynamics and Control. Oxford University Press, Oxford.

2. Berger, R. D. 1981. Comparison of Gompertz and logistic equations to describe plant disease progress. Phytopathology 71:716-719.

3. Berger, R. D. 1989. Description and application of some general models for plant disease epidemics. Pages 125-149 in: Plant Disease Epidemiology. Population Dynamics and Management. Vol. 2. K. J. Leonard and W. E. Fry, eds. McGraw-Hill, New York.

4. Campbell, C. L., and Madden, L. V. 1990. Introduction to Plant Disease Epidemiology. John Wiley \& Sons, New York.

5. Caole, A. J. 1972. The Growth and Structure of Human Populations, a Mathematical Investigation. Princeton University Press, Princeton.

6. Chan, M. S., and Jeger, M. J. 1994. An analytical model of plant virus disease dynamics with roguing and replanting. J. Appl. Ecol. 31:413427.

7. Dayananda, P. W. A., Billard, L., and Chakraborty, S. 1995. Estimation of rate parameter and its relationship with latent and infectious periods in plant disease epidemics. Biometrics 51:284-292.

8. Diekmann, O. 1977. Limiting behaviour in an epidemic model. Nonlinear Analysis, Theory, Methods and Applications 1:459-470.

9. Diekmann, O., and Heesterbeek, J. A. P. 2000. Mathematical Epidemiology of Infectious Diseases. Model Building, Analysis and Interpretation. John Wiley \& Sons, Chichester, England.

10. Diekmann, O., Heesterbeek, J. A. P., and Metz, J. A. J. 1995. The legacy of Kermack and McKendrick. Pages 85-115 in: Epidemic Models: Their Structure and Relation to Data. D. Mollison, ed. Cambridge University Press, Cambridge.

11. Fleming, R. A. 1983. Development of a simple mechanistic model of cereal rust progress. Phytopathology 73:308-312.

12. Heesterbeek, J. A. P., and Dietz, K. 1996. The concept of $R_{0}$ in epidemic theory. Stat. Neerlandica 50:89-110.

13. Jeger, M. J. 1982. The relation between total, infectious, and postinfectious diseased plant tissue. Phytopathology 72:1185-1189.

14. Jeger, M. J. 1984. Relation between rate parameters and latent and infectious periods during a plant disease epidemic. Phytopathology 74:11481152 .

15. Jeger, M. J. 2000. Theory and plant epidemiology. Plant Pathol. 49:651658.

16. Jeger, M. J., and Chan, M. S. 1995. Theoretical aspects of epidemics: Uses of analytical models to make strategic management decisions. Can. J. Plant Pathol. 17:109-114.

17. Jeger, M. J., and van den Bosch, F. 1994. Threshold criteria for model plant disease epidemics. I. Asymptotic results. Phytopathology 84:24-27.

18. Kendall, M. G., and Stuart, A. 1958. The Advanced Theory of Statistics. Vol. 1. Charles Griffin \& Company Ltd., London.

19. Kermack, W. O., and McKendrick, A. G. 1927. A contribution to the mathematical theory of epidemics. Proc. R. Soc. Lond. Ser. A 115:700721.

20. Keyfitz, N. 1968. Introduction to the Mathematics of Population. Addison Wesley, Reading, MA.

21. Keyfitz, N. 1977. Applied Mathematical Demography. Wiley-Interscience, New York.

22. Leonard, K. J., and Mundt, C. C. 1984. Methods for estimating epidemiological effects of quantitative resistance to plant diseases. Theor. Appl. Genet. 67:219-230. 
23. Madden, L. V., and Campbell, C. L. 1990. Nonlinear disease progress curves. Pages 181-229 in: Epidemics of Plant Diseases. J. Kranz, ed. 2nd ed. Springer-Verlag, Berlin.

24. May, R. M. 1990. Population biology and population genetics of plantpathogen associations. Pages 309-325 in: Pests, Pathogens and Plant Communities. J. J. Burdon and S. R. Leather, eds. Blackwell Scientific Publications, Oxford.

25. Metz, J. A. J. 1978. The epidemic in a closed population with all susceptibles equally vulnerable: Some results for large susceptible populations and small initial infections. Acta Biotheor. 27:75-123.

26. Metz, J. A. J., and Diekmann, O. 1986. The Dynamics of Physiologically Structured Populations. Lecture Notes in Biomathematics 68. SpringerVerlag, Berlin.

27. Mollison, D. 1995. The structure of epidemic models. Pages 17-33 in: Epidemic Models: Their Structure and Relation to Data. D. Mollison, ed. Cambridge University Press, Cambridge.

28. Onstad, D. W., and Kornkven, E. A. 1992. Persistence and endemicity of pathogens in plant populations over time and space. Phytopathology 82:561-566.

29. Parlevliet, J. E., and van Ommeren, A. 1975. Partial resistance of barley to leaf rust, Puccinia hordei. 2. Relationship between field trials, microplot tests and latent period. Euphytica 24:293-303.

30. Swinton, J., and Anderson, R. M. 1995. Model frameworks of plant pathogen interactions. Pages 280-294 in: Ecology of Infectious Diseases in Natural Populations. B. T. Grenfell and A. P. Dobson, eds. Cambridge University Press, Cambridge.

31. Teng, P. S. 1985. A comparison of simulation approaches to epidemic modelling. Annu. Rev. Phytopathol. 23:351-379.

32. van den Bosch, F., Frinking, H. D., Metz, J. A. J., and Zadoks, J. C. 1988. Focus expansion in plant disease. III: Two experimental examples. Phytopathology 78:919-925.

33. van den Bosch, F., Zadoks, J. C., and Metz, J. A. J. 1988. Focus expansion in plant disease. I: The constant rate of focus expansion. Phytopathology 78:54-58.

34. van den Bosch, F., Zadoks, J. C., and Metz, J. A. J. 1988. Focus expansion in plant disease. II: Realistic parameter-sparse models. Phytopathology 78:59-64.

35. Vanderplank, J. E. 1963. Plant Diseases: Epidemics and Control. Academic Press, New York.

36. Vanderplank, J. E. 1984. Disease Resistance in Plants. 2nd ed. Academic Press, Orlando.

37. Waggoner, P. E. 1974. Simulation of epidemics. Pages 137-160 in: Epidemics of Plant Diseases: Mathematical Analysis and Modelling. J. Kranz, ed. Springer-Verlag, Berlin.

38. Webb, C. R., Gilligan, C. A., and Asher, M. J. C. 1999. A model for the temporal build-up of Polymyxa betae. Phytopathology 89:30-38. 